\title{
ANÁLISE DA QVT EM CENTROS ACADÊMICOS DE UMA IES PÚBLICA A PARTIR DO EMPREGO DO MÉTODO DE ANÁLISE HIERÁRQUICA (AHP)
}

\author{
Tulio Cremonini Entringer \\ Universidade Estadual do Norte Fluminense Darcy Ribeiro \\ tulio_entringer@hotmail.com \\ André Luís Policani Freitas \\ Universidade Estadual do Norte Fluminense Darcy Ribeiro \\ policani@uenf.br
}

\begin{abstract}
RESUMO
Atualmente, o cenário em que as instituições estão inseridas é marcado pela alta competitividade e fortes mudanças, as quais afetam diretamente a vida do trabalhador. Dessa forma, a constante busca pela sobrevivência e pelo avanço da produtividade das organizações pode ser marcada por diferentes formas de precarização do trabalho, gerando assim, repercussões nocivas aos indivíduos. Nesse contexto, a Qualidade de Vida no Trabalho (QVT) vem ganhando cada vez mais destaque dentro das organizações, bem como nos meios acadêmicos, que visam entender tais situações. Desejando contribuir para esta problemática, este artigo apresenta um estudo experimental, em uma IES pública, com o objetivo de identificar o Centro Acadêmico crítico em termo do grau desempenho da QVT na Universidade, segundo a percepção dos servidores técnico-administrativos, a partir do emprego do Método de Análise Hierárquica (AHP). Como resultados, sugere-se que o Centro de Ciência e Tecnologia (CCT) deve ser priorizado em prol da melhoria da qualidade de vida na IES. Da mesma forma, através dos julgamentos, foi possível observar que a dimensão "Compensação Justa e Adequada”, é o critério de maior preocupação quanto ao grau de desempenho da QVT. Vale destacar que todas as matrizes de julgamentos foram consideradas consistentes.
\end{abstract}

Palavra-chave: Método de Análise Hierárquica; Qualidade de Vida no Trabalho; Instituições de Ensino Superior; Servidores técnico-administrativos.

\begin{abstract}
The current economic scenario has been characterized by high competitiveness and constant changes in organizations, which can directly influence the worker's quality of life. Thus, the constant search for survival and advancement in the productivity of organizations can be marked by different forms of job insecurity, thus generating harmful repercussions for individuals. In this context, Quality of Work Life (QWL) has been gaining more and more prominence within organizations, as well as in academic circles, which aim to understand such situations. Wishing to contribute to this problem, this paper presents an experimental study, in a public University, with the objective of identifying the critical academic center in terms of the degree of QWL performance, according to the perception of the technical-
\end{abstract}


administrative employees, from the application of the Analytic Hierarchy Process (AHP). As a result, it is suggested that the Science and Technology Center (STC) should be prioritized in order to improve the quality of life in the University. Likewise, through the judgments, it was possible to observe that the "Just and Adequate Compensation" dimension is the criterion of greatest concern regarding the degree of QWL performance. It is worth mentioning that all judging matrices were considered consistent.

Keywords: Analytic Hierarchy Process; Quality of Work Life; Universities; Technicaladministrative employees.

\section{Como Citar:}

ENTRINGER, Tulio Cremonini; FREITAS, André Luís Policani. Análise da QVT em centros acadêmicos de uma IES pública a partir do emprego do Método de Análise Hierárquica (AHP). In: SIMPÓSIO DE PESQUISA OPERACIONAL E LOGÍSTICA DA MARINHA, 19., 2019, Rio de Janeiro, RJ. Anais [...]. Rio de Janeiro: Centro de Análises de Sistemas Navais, 2019.

\section{INTRODUÇÃO}

O panorama atual em que as instituições estão inseridas é marcado pela alta competitividade e fortes mudanças, as quais afetam diretamente a vida do trabalhador. Dessa forma, a constante busca pela sobrevivência e pelo avanço da produtividade das organizações pode ser marcada por diferentes formas de precarização do trabalho, gerando assim, repercussões nocivas aos indivíduos (PINTO, 2013). Com isso, neste cenário, a Gestão da Qualidade vem se tornando um dos ramos de atividades mais importantes para as organizações.

Diante do exposto, emerge o desafio de manter o desempenho organizacional e, simultaneamente, disponibilizar condições necessárias para que o trabalhador se sinta satisfeito e motivado com o trabalho. Nesse contexto, a Qualidade de Vida no Trabalho (QVT) vem ganhando cada vez mais destaque dentro das organizações, bem como nos meios acadêmicos, que visam entender esses processos de mudanças (FREITAS et. al, 2013).

A QVT relaciona-se com a mobilização, o comprometimento pessoal e a participação com o bem-estar do funcionário na execução da tarefa, visando o alcance das metas da Qualidade Total (FREITAS; SOUZA, 2009). Sendo assim, um ambiente de trabalho onde há uma gestão eficaz e contingencial de fatores físicos, sociológicos, psicológicos e tecnológicos do próprio trabalho torna-se saudável e mais propício ao aumento de produtividade.

Ao longo dos anos, vários pesquisadores têm se dedicado à elaboração de modelos que buscam compreender e avaliar a QVT sob diversos aspectos, dentre esses se destacam: Walton (1973); Hackman e Oldham (1975); Westley (1979); e Davis e Werther (1983).

Embora a maioria das pesquisas na área da QVT tem sido relacionada a organizações industriais e de serviços (JANES; WISNOM, 2011; MANJUNATH; KURIAN, 2011), tem havido uma atenção crescente acerca da satisfação dos colaboradores nos meios acadêmicos (MIRKAMALI; THANI, 2011). A razão para este interesse, se justifica pelo cenário onde as Instituições de Ensino Superior (IES) estão inseridas. As IES possuem trabalhos intensivos, seus orçamentos são predominantemente destinados com pessoal e sua eficácia é em grande parte dependente da sua equipe (TOKEN, 2011). Nesse cenário, destaca-se o trabalho dos servidores técnico-administrativos nas Universidades. 
Neste contexto, com o intuito de contribuir para esta questão, o presente trabalho apresenta um estudo experimental realizado a partir do emprego do instrumento proposto por Freitas e Souza (2009), após a incorporação de alguns itens de avaliação (ENTRINGER; FREITAS, 2014a, 2014b, 2015), em uma IES pública, com o objetivo de identificar o Centro Acadêmico crítico em termo do grau desempenho da QVT na Universidade, segundo na percepção dos servidores técnico-administrativos. Para tanto, foi aplicado um instrumento de avaliação composto por múltiplos critérios e o emprego do Método de Análise Hierárquica (AHP - Analytic Hierarchy Process).

O presente artigo está organizado da seguinte forma: a seção 2 apresenta uma breve descrição do método AHP, com ênfase nas etapas de estruturação da hierarquia, o cálculo das matrizes de prioridade e a verificação da consistência dos julgamentos. A seção 3 retrata o estudo experimental, abordando a definição dos critérios e alternativas e a coleta de dados. A seção 4 apresenta a análise de dados, abordando o grau de desempenho e grau de importância, julgamentos paritários e cálculo das prioridades médias locais e globais e a verificação da consistência dos julgamentos. E, por fim, na seção 5 são apresentadas as considerações finais referentes ao estudo.

\section{O MÉTODO DE ANÁLISE HIERÁRQUICA}

O método AHP foi proposto por Saaty no início dos anos 80, com a finalidade de selecionar alternativas em processos decisórios que considerem múltiplos critérios. Adicionalmente, o método permite a hierarquização das alternativas à luz de todos os critérios e subcritérios (se houver), assim como a formação de hierarquias dos subcritérios segundo os critérios. Dentre diversos métodos de apoio à decisão, como diagrama de árvore, teoria dos jogos, programação linear, entre outros, o método AHP tem sido considerado como o mais reconhecido e utilizado (VELASCO; FREITAS, 2014). .

Diversas pesquisas têm utilizado o AHP como ferramenta de apoio à decisão em diversas áreas de conhecimento, como por exemplo: classificação de inventário (KEREN; HADAD, 2016), avaliação da qualidade de água subterrânea (KAVURMACI; ÜSTÜN, 2016), avaliação do desempenho do ensino superior (THANASSOULIS et al., 2017), mensuração da qualidade do serviço de saúde (SINGH; PRASHER, 2017), entre outros.

De acordo com Saaty (1990), e corroborado por Goyal, Rahman e Kazmi (2015), o método AHP se divide em cinco etapas: (i) definição dos critérios, subcritérios e as alternativas; (ii) aquisição dos julgamentos comparativos; (iii) avaliação das alternativas em relação aos critérios; (iv) determinação das prioridades médias (dos critérios e subcritérios); determinação das prioridades globais (dos critérios em relação às alternativas); e (v) verificação das consistências dos julgamentos.

\subsection{ESTRUTURA HIERÁRQUICA}

O funcionamento do método AHP está estruturado na hierarquia composta por vários níveis de informações: objetivo geral (ou foco principal); critérios (ou entidades); subcritérios (se existirem), e no alicerce da estrutura, as alternativas.

Saaty (1990) define hierarquia como um sistema particular, baseado no conceito de que as entidades identificadas, podem ser agrupadas em conjuntos distintos. $\mathrm{O}$ autor afirma ainda que, os elementos de cada nível são independentes entre si. Além disso, é importante ressaltar que na maioria dos casos as alternativas recebem influências dos critérios, mas é possível que, em alguns desses casos, os critérios também influenciem as alternativas, fenômeno denominado de "impacto inverso". Quando uma hierarquia é bem arquitetada, ela demonstra bem a realidade, mesmo desconsiderando os impactos inversos. A Figura 1 demonstra a estrutura básica de uma hierarquia. 
Figura 1- Estrutura básica de uma hierarquia

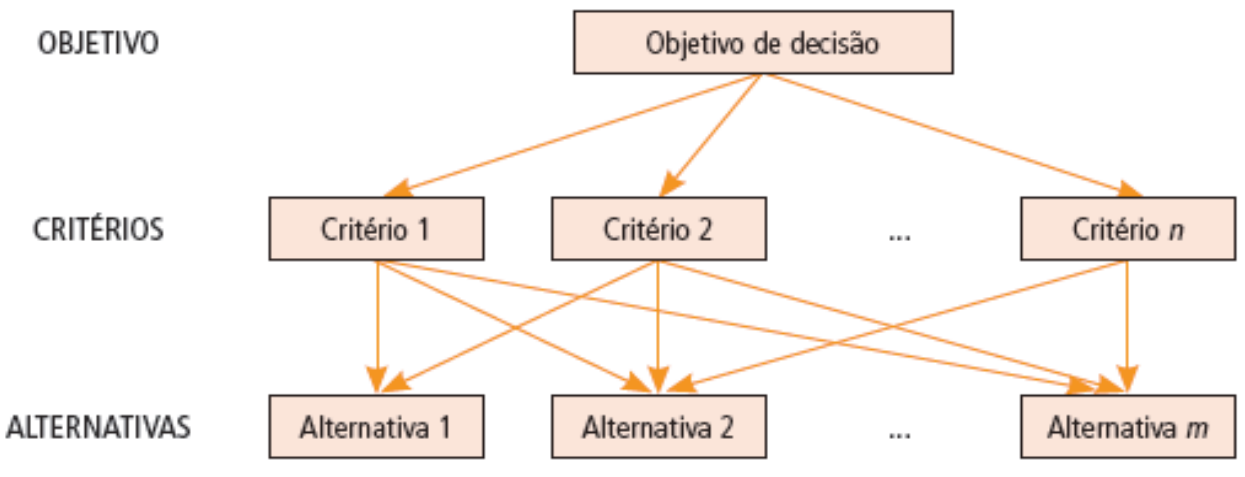

Fonte: Adaptado de Saaty (1991)

Modelar um problema de forma hierárquica é bastante adequado, uma vez que permite uma visão bastante ampla de todo o sistema, e possibilita que as influências entre os diversos elementos sejam facilmente identificadas. Esta estrutura ajuda o decisor a examinar toda a complexidade do problema, além de ver partes deste. Através disso, é possível o decisor conseguir prioridades por meio da comparação par a par, feita pelo usuário (LAÑEZ; CUNHA, 2006).

Para Saaty (1990), a vantagem da estrutura hierárquica é poder compreender o entrosamento dos níveis mais elevados a partir das influências mútuas entre os diversos níveis existentes na mesma hierarquia.

\subsection{PRIORIDADE DAS HIERARQUIAS}

Posteriormente a estruturação do problema, se faz necessário encontrar as prioridades dos elementos de um nível em relação aos de outro nível obtendo os julgamentos dos usuários feito através da comparação par a par, que resultará numa matriz de decisão para cada critério ou subcritério e, dos critérios em relação ao foco principal. Para se construir uma matriz de decisão, é necessária uma quantidade " $x x$ ” de julgamentos, que é dada pela Equação 1:

$$
x=\frac{n(n-1)}{2}
$$

onde $\mathrm{n} n$ é a ordem da matriz. Nesta etapa, o julgador irá avaliar sua preferência entre os fatores, considerando os critérios no nível superior. Para tanto, é preciso uma escala de conversão, para atribuir valores aos seus julgamentos.

A Tabela 1 apresenta a escala sugerida por Saaty (2000), conhecida na literatura como escala fundamental de Saaty.

Tabela 1 - Escala fundamental de Saaty

\begin{tabular}{c|c|l}
\hline $\begin{array}{c}\text { Escala } \\
\text { Numérica }\end{array}$ & $\begin{array}{c}\text { Escala } \\
\text { Conceitual }\end{array}$ & \multicolumn{1}{c}{ Descrição } \\
\hline 1 & Igual & Os dois elementos comparados contribuem igualmente para o objetivo \\
\hline 3 & Moderada & Os elementos comparado é ligeiramente importante ao outro \\
\hline 5 & Forte & A experiência e o julgamento favorecem fortemente o elemento em relação ao outro \\
\hline 7 & Muito Forte & $\begin{array}{l}\text { O elemento comparado é muito mais forte em relação ao outro, e tal importância } \\
\text { pode ser observada na prática }\end{array}$ \\
\hline 9 & Absoluta & O elemento comparado apresenta o mais alto nível de evidência possível a seu favor \\
\hline $2 / 4 / 6 / 8$ & \multicolumn{2}{c}{ Valores intermediários entre dois julgamentos, utilizados quando o decisor sentir dificuldade ao } \\
escolher entre dois graus de importância adjacentes.
\end{tabular}


Fonte: Adaptado de Saaty (2000)

As matrizes de julgamento devem ser normalizadas a fim de obter as prioridades médias locais e globais. Saaty (1990) destaca vários métodos para a normalização da matriz. Em um desses, somam-se os valores das colunas da matriz, depois se divide cada elemento da matriz pela soma dos valores da sua própria coluna, o que resultará no quadro normalizado. A normalização da matriz resultará numa outra matriz, e para se encontrar as prioridades médias locais (PML’s) é necessário somar as linhas da nova matriz e dividir pelo número de colunas. Para se encontrar as prioridades globais (PG's) multiplicam-se as PML's dos subcritérios à luz das alternativas pelas PML's dos critérios em relação ao foco principal. Este cálculo é repetido com todos os critérios e alternativas da mesma linha (TREVIZANO; FREITAS, 2005).

\subsection{CONSISTÊNCIA DOS JULGAMENTOS}

Costa (2002) ressalta que os julgamentos paritários estão fundamentados na experiência e conhecimento de profissionais, entretanto, inconsistências podem ocorrer principalmente quando existir um grande número de julgamentos. Desta forma, o AHP propõe calcular a Razão de Consistência (RC), que diz respeito à avaliação da inconsistência em função da ordem da matriz de julgamentos. A Razão de Consistência é calculada pela Equação 2:

$$
R C=I C / I R
$$

onde IC é o Índice de Consistência que é dado por $I C=\left(\lambda_{\text {máx }}-n\right) /(n-1)$ e $\lambda_{\text {máx }}$ é o maior autovalor da matriz de julgamentos de ordem n. O valor de $I R$, Índice de Consistência Randômica, é fixo e tem como base o número de critérios que está sendo avaliado (Quadro 1). A Razão de Consistência será aceita se $R C \leq 0,10$ (SAATY, 1990).

\begin{tabular}{|c|c|c|c|c|c|c|c|c|c|c|c|c|}
\hline \multicolumn{13}{|c|}{ Quadro 1 - Índices de consistência randômicos (IR) } \\
\hline$n$ & 1 & 2 & 3 & 4 & 5 & 6 & 7 & 8 & 9 & 10 & 11 & 12 \\
\hline$I R$ & 0 & 0 & 0,58 & 0,9 & 1,12 & 1,24 & 1,32 & 1,41 & 1,45 & 1,49 & 1,51 & 1,54 \\
\hline
\end{tabular}

Fonte: Adaptado de Costa (2002)

Segundo Lañez e Cunha (2006), o conceito de consistência é oferecido através de uma quantidade de dados que são apresentados no início do processo e, a partir deste, é possível, por meio da lógica, deduzir todos os outros dados. A falta de consistência nos julgamentos não afirma que o método é falho, mas “normal e útil”, desde que controlada.

\section{O ESTUDO EXPERIMENTAL}

\subsection{DEFINIÇÃO DE CRITÉRIOS}

Foram considerados 28 itens (subcritérios) agrupados em 7 dimensões (critérios) referentes ao grau de desempenho da IES em termos de QVT (FREITAS. SOUZA, 2009). O Quadro 2 apresenta, de forma resumida, a descrição das 'Dimensões' e aspectos abordados, enquanto o Quadro 3 mostra os itens referentes à cada critério (dimensão), formulados a partir dos itens de avaliação do instrumento proposto por Freitas e Souza (2009), após a incorporação de alguns itens de avaliação (ENTRINGER; FREITAS, 2014a, 2014b, 2015).

Quadro 2 - Descrição das dimensões e aspectos abordados no instrumento de pesquisa

\begin{tabular}{|l|l|}
\hline \multicolumn{1}{|c|}{ Dimensões } & \multicolumn{1}{|c|}{ Aspecto considerado } \\
\hline $\begin{array}{l}\text { D1. Integração } \\
\text { social na }\end{array}$ & $\begin{array}{l}\text { Relacionamento pessoal entre pares no mesmo departamento, com outros } \\
\text { departamentos e com superiores em termos de igualitarismo, ausência de preconceitos, }\end{array}$ \\
\hline
\end{tabular}




\begin{tabular}{|l|l|}
\hline organização & facilidade de mobilidade social, apoio social, e condições de abertura interpessoal. \\
\hline $\begin{array}{l}\text { D2. Utilização da } \\
\text { capacidade humana }\end{array}$ & $\begin{array}{l}\text { Condições de uso da capacidade do servidor em termos de autonomia, uso de } \\
\text { habilidades múltiplas, quantidade e qualidade de informações recebidas, execução de } \\
\text { tarefas completas, planejamento do trabalho, e feedback (comentários a respeito do } \\
\text { trabalho realizado). }\end{array}$ \\
\hline $\begin{array}{l}\text { D3. Segurança e } \\
\text { saúde nas condições } \\
\text { de trabalho }\end{array}$ & $\begin{array}{l}\text { Condições físicas, ambientais e psicológicas que podem afetar o bem-estar do servidor } \\
\text { em termos de: horários; condições de segurança; limites de idade para o trabalho; } \\
\text { qualidade e quantidade de recursos materiais, e; condições psicológicas. }\end{array}$ \\
\hline $\begin{array}{l}\text { D4. } \\
\text { Constitucionalismo }\end{array}$ & $\begin{array}{l}\text { Direitos e deveres do servidor em termos de privacidade, livre expressão, igualdade de } \\
\text { tratamento e possibilidade de recurso a decisões da organização. }\end{array}$ \\
\hline $\begin{array}{l}\text { D5. Trabalho e } \\
\text { espaço de vida }\end{array}$ & Horas extras, tempo com a família, e realização de atividades cívicas beneficentes. \\
\hline $\begin{array}{l}\text { D6. Compensação } \\
\text { justa e adequada }\end{array}$ & $\begin{array}{l}\text { Relação entre o pagamento e a contribuição do servidor para com a organização } \\
\text { (esforço, experiência, habilidades e qualificações) e relação existente entre o salário e } \\
\text { o cargo ocupado. }\end{array}$ \\
\hline $\begin{array}{l}\text { D7. Relevância } \\
\text { Social do trabalho }\end{array}$ & $\begin{array}{l}\text { Prestígio obtido pela organização por ser socialmente responsável e pelos benefícios } \\
\text { proporcionados à comunidade na qual está inserida. }\end{array}$ \\
\hline
\end{tabular}
Fonte: Freitas e Souza (2009)

Quadro 3-Definição de critérios e subcritérios para a mensuração da QVT segundo o desempenho da IES

\begin{tabular}{|c|c|}
\hline Crit. & Subcritério \\
\hline \multirow{7}{*}{ D1 } & $\mathbf{P}_{1}$. Relacionamento com os funcionários do mesmo nível hierárquico \\
\hline & $\mathbf{P}_{2 .}$ Relacionamento com seu superior \\
\hline & $\mathbf{P}_{3}$. Relacionamento com seus subalternos \\
\hline & $\mathbf{P}_{4}$. Relacionamento com funcionários de outros setores ou departamentos \\
\hline & $\mathbf{P}_{5 .}$ Cooperação entre os colegas de setor \\
\hline & $\mathbf{P}_{6}$. Interação com outras pessoas, requerido para execução do trabalho \\
\hline & $\mathbf{P}_{7}$. Ponto de vista dos colegas de setor quando um membro do grupo é promovido como o líder deste \\
\hline \multirow{7}{*}{ D2 } & $\mathbf{P}_{8 .}$ Observações construtivas de seu superior sobre o trabalho \\
\hline & 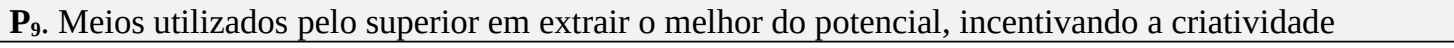 \\
\hline & $\mathbf{P}_{\mathbf{1 0}}$. Independência na realização das atividades dentro da empresa \\
\hline & $\mathbf{P}_{11 .}$ Participação, permitido pela organização, no planejamento do próprio trabalho \\
\hline & $\begin{array}{l}\mathbf{P}_{12} \text {. Grau em que o trabalho envolve tarefas complexas, ou seja, que contém em si outras atividades } \\
\text { utilizando a variedade de conhecimento, habilidades e talentos }\end{array}$ \\
\hline & $\mathbf{P}_{\mathbf{1 3}}$ Quantidade de informações acessíveis para a realização do trabalho \\
\hline & $\mathbf{P}_{\mathbf{1 4}}$. Qualidade das informações acessíveis para a realização do trabalho \\
\hline \multirow{5}{*}{ D3 } & $\mathbf{P}_{15 .}$ Condições ambientais de trabalho (iluminação, higiene, ventilação e organização) para a realização do trabalho \\
\hline & $\mathbf{P}_{\mathbf{1 6} .}$ Quantidade de recursos materiais, necessários, oferecidos para execução da função \\
\hline & $\mathbf{P}_{\mathbf{1 7} .}$ Qualidade de recursos disponíveis para execução da função \\
\hline & $\mathbf{P}_{\mathbf{1 8}}$. Acessibilidade do local de trabalho \\
\hline & $\mathbf{P}_{\mathbf{1 9}}$. Área do local de trabalho (espaço suficiente para todos) \\
\hline \multirow{2}{*}{ D4 } & $\mathbf{P}_{\mathbf{2 0}}$. Preservação da privacidade dentro da organização \\
\hline & $\mathbf{P}_{21}$. Possibilidade de revisão das decisões tomadas na organização, ou seja, de recurso \\
\hline \multirow[b]{2}{*}{ D5 } & $\mathbf{P}_{22 .}$ Tempo gasto com a família, sem ser afetado pelo trabalho levado para concluir em casa \\
\hline & $\begin{array}{l}\mathbf{P}_{23} \text { Impacto causado pela atividade exercida na empresa na vida de outras pessoas dentro ou fora da } \\
\text { organização }\end{array}$ \\
\hline \multirow{3}{*}{ D6 } & $\begin{array}{l}\text { P }_{24 .} \text { Valor do pagamento em relação a sua contribuição (esforço, experiência, habilidades e } \\
\text { qualificação) para a organização }\end{array}$ \\
\hline & $\mathbf{P}_{25}$. Benefícios recebidos (vale transporte, auxílio alimentação, auxílio creche, assistência médica e odontológica) \\
\hline & $\begin{array}{l}\mathbf{P}_{26 .} \text { Valor do salário em relação ao cargo ocupado, quando comparado ao valor pago por este mesmo } \\
\text { cargo em outras organizações do mesmo ramo }\end{array}$ \\
\hline \multirow{2}{*}{ D7 } & $\mathbf{P}_{27}$, Importância que o trabalho tem na vida \\
\hline & $\mathbf{P}_{\mathbf{2 8}}$ Imagem da Universidade perante a comunidade local \\
\hline
\end{tabular}

Fonte: autoria própria (2019) 


\subsection{DEFINIÇÃO DE ALTERNATIVAS}

As alternativas utilizadas para a avaliação da QVT, segundo o grau de desempenho da IES são os Centros Acadêmicos presentes na IES pública em questão: Centro de Ciências e Tecnologia (CCT); Centro de Ciências e Tecnologias Agropecuárias (CCTA); Centro de Ciências do Homem (CCH); Centro de Biociências e Biotecnologia (CBB); e Reitoria. A Figura 2 apresenta a hierarquia resultante deste estudo.

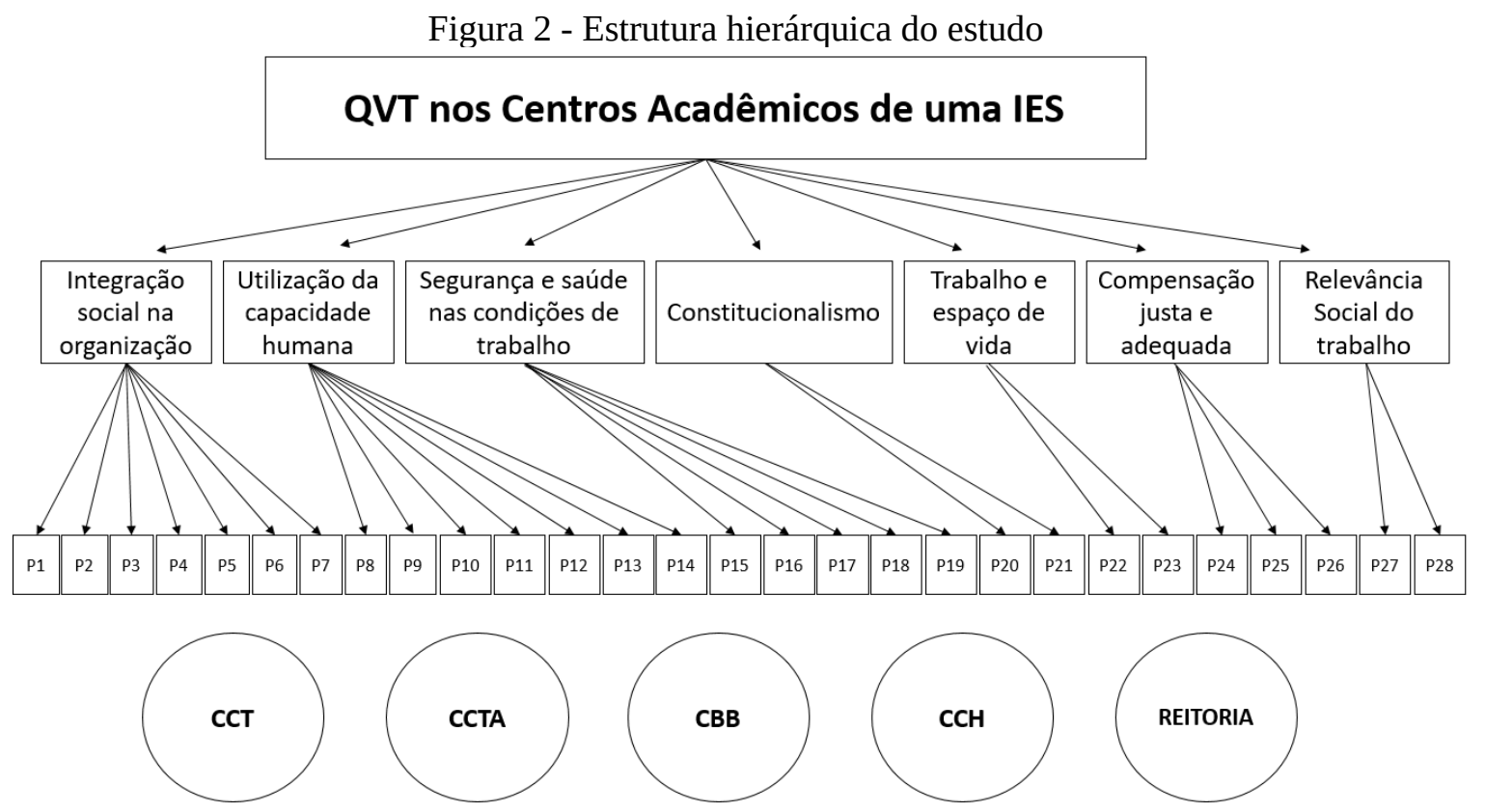

Fonte: autoria própria (2019)

\subsection{COLETA DE DADOS}

A aplicação do questionário ocorreu no primeiro semestre letivo de 2014, com a participação de 277 servidores técnicos-administrativos respondentes, como exposto no Tabela 2. O Gráfico 1, mostra a distribuição do número de questionários respondidos por Centro em relação ao número total de respondentes na IES.

Tabela 2 - Distribuição do número de questionários respondidos por Centro

\begin{tabular}{cccc}
\hline Centro & $\begin{array}{c}\text { Número de } \\
\text { servidores }\end{array}$ & Número de respondentes & $\begin{array}{c}\text { Porcentagem do tamanho } \\
\text { amostral }\end{array}$ \\
\hline CCT & 66 & 34 & $52 \%$ \\
CCTA & 166 & 90 & $54 \%$ \\
CCH & 37 & 24 & $65 \%$ \\
CBB & 71 & 37 & $52 \%$ \\
Reitoria & 194 & 92 & $47 \%$ \\
\hline IES & $\mathbf{5 3 4}$ & $\mathbf{2 7 7}$ & $\mathbf{5 2 \%}$ \\
\hline
\end{tabular}

Fonte: autoria própria (2019) 
Gráfico 1 - Distribuição do número de questionários respondidos por Centro em relação ao número total de respondentes na IES

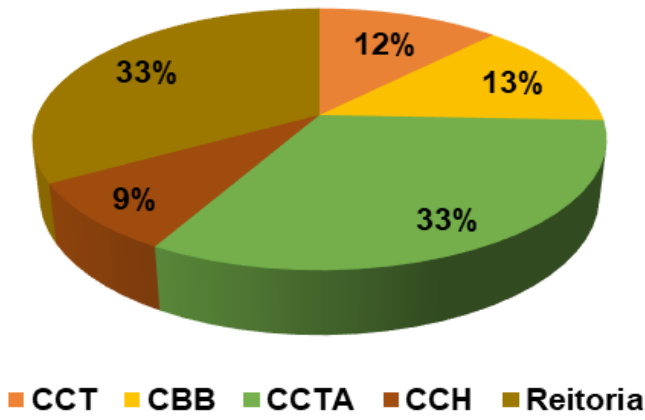

Fonte: autoria própria (2019)

Por meio de um questionário, presente no Anexo I, os itens (subcritérios) foram analisados pelos respondentes, que eram solicitados a avaliar a QVT segundo o grau de desempenho da IES. Para tanto, foram consideradas uma escala de mensuração com 5 pontos, não-forçadas e balanceadas (Quadro 4).

Quadro 4 - Escalas utilizadas para avaliação da QVT

\begin{tabular}{|c|c|}
\hline QVT segundo o desempenho da IES & Valor \\
\hline Muito Ruim & 1 \\
\hline Ruim & 2 \\
\hline Neutro & 3 \\
\hline Bom & 4 \\
\hline Muito Bom & 5 \\
\hline
\end{tabular}

Fonte: Adaptado de Freitas, Souza e Quintella (2013).

As alternativas utilizadas para a avaliação da QVT, segundo o grau de desempenho da IES são os Centros Acadêmicos presentes na IES pública em questão: Centro de Ciências e Tecnologia (CCT); Centro de Ciências e Tecnologias Agropecuárias (CCTA); Centro de Ciências do Homem (CCH); Centro de Biociências e Biotecnologia (CBB); e Reitoria. A Figura 2 apresenta a hierarquia resultante deste estudo.

\section{ANÁLISE DE DADOS}

\subsection{GRAU DE DESEMPENHO E GRAU DE IMPORTÂNCIA}

O Quadro 5 apresenta o grau de desempenho da IES, em termos de QVT, obtidos a partir dos julgamentos dos servidores técnicos-administrativos lotados nos Centros Acadêmicos de uma Universidade Pública e utilizados como dados para o emprego do AHP.

Na ausência de julgamentos referentes ao grau de importância, decidiu-se encontrálos, com base no grau de desempenho, a partir dos elementos de um nível em relação aos de outro nível obtendo os julgamentos dos usuários feito através da comparação par a par. Para tanto, é necessário realizar uma conversão de escalas (escala de Saaty), para atribuir valores aos seus julgamentos. É importante destacar que, na conversão para a escala de Saaty, o grau de importância possui sentido inverso, uma vez que, em termos de melhoria da qualidade, a maior importância deve ser dada aos critérios de pior desempenho, uma vez que se busca o Centro Acadêmico crítico em termo de QVT. 


\begin{tabular}{|c|c|c|c|c|c|c|c|c|c|c|c|}
\hline \multirow[b]{3}{*}{ Critério } & \multirow[b]{3}{*}{ Subcritérios } & \multicolumn{10}{|c|}{ Grau de desempenho } \\
\hline & & \multicolumn{2}{|c|}{ CCT } & \multicolumn{2}{|c|}{ CCTA } & \multicolumn{2}{|c|}{ CBB } & \multicolumn{2}{|c|}{$\mathrm{CCH}$} & \multicolumn{2}{|c|}{ REITORIA } \\
\hline & & $Q V T$ & $\overline{Q V T}$ & $Q V T$ & $\overline{Q V T}$ & QVT & $\overline{Q V T}$ & $Q V T$ & $\overline{Q V T}$ & $Q V T$ & $\overline{Q V T}$ \\
\hline \multirow{7}{*}{ Dl } & $\mathbf{P}_{1}$ & 4,00 & \multirow{7}{*}{3,73} & 4,14 & \multirow{7}{*}{3,94} & 3,94 & \multirow{7}{*}{3,87} & 4,38 & \multirow{7}{*}{4,09} & 4,40 & \multirow{7}{*}{4,18} \\
\hline & $\mathbf{P}_{2}$ & 4,10 & & 4,06 & & 4,03 & & 4,21 & & 4,22 & \\
\hline & $P_{3}$ & 4,13 & & 4,06 & & 4,09 & & 4,38 & & 4,34 & \\
\hline & $\mathbf{P}_{4}$ & 3,74 & & 3,93 & & 3,81 & & 4,13 & & 4,04 & \\
\hline & $\mathbf{P}$ & 3,42 & & 3,82 & & 3,83 & & 3,96 & & 4,14 & \\
\hline & $\mathbf{P}_{6}$ & 3,45 & & 3,89 & & 3,75 & & 3,96 & & 4,13 & \\
\hline & $\mathbf{P}_{7}$ & 3,29 & & 3,66 & & 3,64 & & 3,65 & & 3,99 & \\
\hline \multirow{7}{*}{ D2 } & $\mathbf{P}_{8}$ & 4,06 & \multirow{7}{*}{3,71} & 4,11 & \multirow{7}{*}{3,85} & 3,97 & \multirow{7}{*}{3,81} & 4,22 & \multirow{7}{*}{3,84} & 4,04 & \multirow{7}{*}{3,81} \\
\hline & $P_{9}$ & 3,65 & & 3,81 & & 3,60 & & 3,52 & & 3,33 & \\
\hline & $\mathbf{P}_{10}$ & 3,84 & & 3,80 & & 3,77 & & 4,00 & & 4,04 & \\
\hline & $\mathbf{P}_{11}$ & 3,83 & & 3,85 & & 3,83 & & 3,78 & & 3,96 & \\
\hline & $\mathbf{P}_{12}$ & 3,71 & & 3,70 & & 3,74 & & 3,70 & & 3,87 & \\
\hline & $\mathbf{P}_{13}$ & 3,55 & & 3,89 & & 3,94 & & 3,96 & & 3,73 & \\
\hline & $\mathbf{P}_{14}$ & 3,35 & & 3,76 & & 3,83 & & 3,68 & & 3,69 & \\
\hline \multirow{5}{*}{ D3 } & $\mathbf{P}_{15}$ & 2,61 & \multirow{5}{*}{2,88} & 3,17 & \multirow{5}{*}{3,29} & 3,08 & \multirow{5}{*}{3,28} & 3,52 & \multirow{5}{*}{3,51} & 3,61 & \multirow{5}{*}{3,35} \\
\hline & $\mathbf{P}_{16}$ & 3,10 & & 3,75 & & 3,44 & & 3,57 & & 3,35 & \\
\hline & $\mathbf{P}_{17}$ & 3,13 & & 3,70 & & 3,47 & & 3,39 & & 3,23 & \\
\hline & $\mathbf{P}_{18}$ & 2,45 & & 2,69 & & 3,00 & & 3,40 & & 2,96 & \\
\hline & $\mathbf{P}_{19}$ & 3,13 & & 3,15 & & 3,39 & & 3,70 & & 3,59 & \\
\hline \multirow{2}{*}{ D4 } & $\mathbf{P}_{20}$ & 3,06 & \multirow{2}{*}{2,77} & 3,51 & 338 & 3,46 & 327 & 3,87 & 348 & 3,51 & 322 \\
\hline & $\mathbf{P}_{21}$ & 2,48 & & 3,25 & & 3,09 & & 3,10 & $3,+0$ & 3,02 & 3,21 \\
\hline DE & $\mathbf{P}_{22}$ & 3,58 & 345 & 3,90 & 388 & 4,03 & 300 & 4,00 & 303 & 4,02 & 380 \\
\hline Ds & $\mathbf{P}_{23}$ & 3,32 & & 3,86 & & 3,94 & & 3,87 & & 3,57 & \\
\hline & $\mathbf{P}_{24}$ & 2,52 & & 2,66 & & 2,66 & & 2,65 & & 2,47 & \\
\hline D6 & $\mathbf{P}_{25}$ & 2,32 & 2,41 & 2,26 & 2,42 & 2,43 & 2,43 & 2,46 & 2,52 & 2,39 & 2,44 \\
\hline & $\mathbf{P}_{26}$ & 2,39 & & 2,34 & & 2,20 & & 2,43 & & 2,46 & \\
\hline D7 & $\mathbf{P}_{27}$ & 4,23 & 406 & 4,20 & 417 & 4,25 & 425 & 4,04 & 424 & 4,15 & 423 \\
\hline $\mathbf{D}]$ & $\mathbf{P}_{28}$ & 3,90 & $t, 00$ & 4,14 & 7,17 & 4,25 & 4,23 & 4,43 & $-7,2+$ & 4,31 & 4,25 \\
\hline
\end{tabular}

Fonte: autoria própria (2019)

\subsection{JULGAMENTOS PARITÁRIOS E CÁLCULO DAS PRIORIDADES MÉDIAS LOCAIS}

Na Tabela 3 estão os julgamentos paritários em relação ao foco principal e as prioridades médias locais (PML), obtidas por meio do emprego do software IPÊ (COSTA, 2004), que implementa a lógica do método AHP. É possível observar que a dimensão Compensação Justa e Adequada (D6), é o critério mais importante, em termos do grau de desempenho da QVT na IES, segundo a percepção dos servidores técnicos-administrativos.

Tabela 3 - Matrizes de julgamentos paritários dos critérios à luz do Foco Principal

\begin{tabular}{ccccccccc}
\hline Foco Principal & D1 & D2 & D3 & D4 & D5 & D6 & D7 & PML \\
\hline D1 & 1 & 1 & $1 / 4$ & $1 / 4$ & 1 & $1 / 8$ & 2 & 0,054 \\
D2 & 1 & 1 & $1 / 3$ & $1 / 3$ & 1 & $1 / 7$ & 2 & 0,058 \\
D3 & 4 & 3 & 1 & 1 & 3 & $1 / 5$ & 5 & 0,159 \\
D4 & 4 & 3 & 1 & 1 & 3 & $1 / 5$ & 5 & 0,159 \\
D5 & 1 & 1 & $1 / 3$ & $1 / 3$ & 1 & $1 / 7$ & 2 & 0,058 \\
D6 & 8 & 7 & 5 & 5 & 7 & 1 & 9 & $\mathbf{0 , 4 8 0}$ \\
D7 & $1 / 2$ & $1 / 2$ & $1 / 5$ & $1 / 5$ & $1 / 2$ & $1 / 9$ & 1 & 0,033 \\
\hline
\end{tabular}

Fonte: autoria própria (2019) 
O mesmo procedimento foi realizado com os subcritérios em relação aos respectivos critérios, conforme mostra a Tabela 4. Por meio desta tabela, é possível constatar quais subcritérios são prioritários à luz de cada critério, ou seja, o Centro que apresenta maior PML.

Tabela 4 - Matrizes de julgamentos paritários dos subcritérios à luz dos critérios

\begin{tabular}{|c|c|c|c|c|c|c|c|c|}
\hline D1 & P1 & P2 & P3 & P4 & P5 & P6 & P7 & PML \\
\hline P1 & 1 & 1 & 1 & $1 / 2$ & $1 / 2$ & $1 / 2$ & $1 / 3$ & 0,084 \\
\hline P2 & 1 & 1 & 1 & 1 & $1 / 2$ & $1 / 2$ & $1 / 3$ & 0,094 \\
\hline P3 & 1 & 1 & 1 & $1 / 2$ & $1 / 2$ & $1 / 2$ & $1 / 3$ & 0,084 \\
\hline P4 & 2 & 1 & 2 & 1 & 1 & 1 & $1 / 2$ & 0,149 \\
\hline P5 & 2 & 2 & 2 & 1 & 1 & 1 & 1 & 0,178 \\
\hline P6 & 2 & 2 & 2 & 1 & 1 & 1 & 1 & 0,178 \\
\hline P7 & 3 & 3 & 3 & 2 & 1 & 1 & 1 & $\mathbf{0 , 2 3 5}$ \\
\hline D2 & P8 & P9 & P10 & P11 & P12 & P13 & P14 & PML \\
\hline P8 & 1 & $1 / 3$ & 1 & 1 & $1 / 2$ & $1 / 2$ & $1 / 2$ & 0,089 \\
\hline P9 & 3 & 1 & 2 & 2 & 1 & 2 & 1 & 0,219 \\
\hline P10 & 1 & $1 / 2$ & 1 & 1 & 1 & 1 & 1 & 0,125 \\
\hline P11 & 1 & $1 / 2$ & 1 & 1 & 1 & 1 & 1 & 0,125 \\
\hline P12 & 2 & 1 & 1 & 1 & 1 & 1 & 1 & 0,152 \\
\hline P13 & 2 & $1 / 2$ & 1 & 1 & 1 & 1 & 1 & 0,137 \\
\hline P14 & 2 & 1 & 1 & 1 & 1 & 1 & 1 & 0,152 \\
\hline D3 & P15 & P16 & P17 & P18 & P19 & & & PML \\
\hline P15 & 1 & 2 & 1 & $1 / 3$ & 1 & & & 0,162 \\
\hline P16 & $1 / 2$ & 1 & 1 & $1 / 4$ & 1 & & & 0,117 \\
\hline P17 & 1 & 1 & 1 & $1 / 3$ & 1 & & & 0,140 \\
\hline P18 & 3 & 4 & 3 & 1 & 3 & & & 0,442 \\
\hline P19 & 1 & 1 & 1 & $1 / 3$ & 1 & & & 0,140 \\
\hline D4 & P20 & P21 & & & & & & PML \\
\hline P20 & 1 & $1 / 3$ & & & & & & 0,250 \\
\hline P21 & 3 & 1 & & & & & & $\mathbf{0 , 7 5 0}$ \\
\hline D5 & P22 & P23 & & & & & & PML \\
\hline P22 & 1 & $1 / 2$ & & & & & & 0,333 \\
\hline P23 & 2 & 1 & & & & & & 0,667 \\
\hline D6 & P24 & P25 & P26 & & & & & PML \\
\hline P24 & 1 & $1 / 2$ & $1 / 2$ & & & & & 0,200 \\
\hline P25 & 2 & 1 & 1 & & & & & $\mathbf{0 , 4 0 0}$ \\
\hline P26 & 2 & 1 & 1 & & & & & $\mathbf{0 , 4 0 0}$ \\
\hline D7 & $\mathrm{P} 27$ & P28 & & & & & & PML \\
\hline P27 & 1 & 1 & & & & & & $\mathbf{0 , 5 0 0}$ \\
\hline P28 & 1 & 1 & & & & & & $\mathbf{0 , 5 0 0}$ \\
\hline
\end{tabular}

Fonte: autoria própria (2019)

Também, foram realizados julgamentos paritários das alternativas em relação aos 28 subcritérios considerados, e as PML's de cada um destes subcritérios estão expostos na Tabela 5. 
Tabela 5 - Prioridades médias locais a partir dos julgamentos paritários das alternativas à luz dos subcritérios

\begin{tabular}{cccccc}
\hline Alternativas & CCT & CCTA & CCH & CBB & REITORIA \\
\hline Subcritério & & & PML's & & \\
\hline P1 & 0,261 & 0,241 & 0,111 & $\mathbf{0 , 2 8 3}$ & 0,103 \\
P2 & 0,200 & 0,200 & 0,200 & 0,200 & 0,200 \\
P3 & $\mathbf{0 , 2 5 0}$ & $\mathbf{0 , 2 5 0}$ & 0,125 & $\mathbf{0 , 2 5 0}$ & 0,125 \\
P4 & $\mathbf{0 , 2 5 4}$ & 0,197 & 0,147 & $\mathbf{0 , 2 5 4}$ & 0,147 \\
P5 & $\mathbf{0 , 4 1 4}$ & 0,177 & 0,143 & 0,163 & 0,103 \\
P6 & $\mathbf{0 , 4 1 0}$ & 0,163 & 0,123 & 0,202 & 0,101 \\
P7 & $\mathbf{0 , 3 6 4}$ & 0,182 & 0,182 & 0,182 & 0,091 \\
\hline P8 & 0,198 & 0,198 & 0,176 & $\mathbf{0 , 2 3 1}$ & 0,198 \\
P9 & 0,166 & 0,116 & 0,219 & 0,189 & $\mathbf{0 , 3 1 1}$ \\
P10 & 0,197 & 0,254 & 0,147 & $\mathbf{0 , 2 5 4}$ & 0,147 \\
P11 & 0,200 & 0,200 & 0,200 & 0,200 & 0,200 \\
P12 & 0,200 & 0,200 & 0,200 & 0,200 & 0,200 \\
P13 & $\mathbf{0 , 3 0 9}$ & 0,167 & 0,132 & 0,142 & 0,250 \\
P14 & $\mathbf{0 , 3 8 0}$ & 0,149 & 0,161 & 0,149 & 0,161 \\
\hline P15 & $\mathbf{0 , 4 6 6}$ & 0,181 & 0,080 & 0,198 & 0,075 \\
P16 & $\mathbf{0 , 3 8 1}$ & 0,095 & 0,126 & 0,178 & 0,221 \\
P17 & $\mathbf{0 , 3 0 1}$ & 0,089 & 0,186 & 0,160 & 0,265 \\
P18 & $\mathbf{0 , 4 0 5}$ & 0,248 & 0,059 & 0,144 & 0,144 \\
P19 & $\mathbf{0 , 3 1 5}$ & 0,315 & 0,100 & 0,156 & 0,115 \\
\hline P20 & $\mathbf{0 , 4 2 1}$ & 0,158 & 0,076 & 0,187 & 0,158 \\
P21 & $\mathbf{0 , 4 7 7}$ & 0,112 & 0,127 & 0,127 & 0,157 \\
\hline P22 & $\mathbf{0 , 4 0 4}$ & 0,159 & 0,146 & 0,146 & 0,146 \\
P23 & $\mathbf{0 , 4 0 6}$ & 0,121 & 0,121 & 0,164 & 0,188 \\
\hline P24 & 0,200 & 0,200 & 0,200 & 0,200 & 0,200 \\
P25 & 0,200 & 0,200 & 0,200 & 0,200 & 0,200 \\
P26 & 0,197 & 0,197 & 0,172 & $\mathbf{0 , 2 6 3}$ & 0,172 \\
\hline P27 & 0,198 & 0,198 & $\mathbf{0 , 2 3 1}$ & 0,176 & 0,198 \\
P28 & $\mathbf{0 , 3 7 5}$ & 0,186 & 0,130 & 0,161 & 0,148 \\
\hline & & F 39 \\
\hline
\end{tabular}

Fonte: autoria própria (2019)

\subsection{CÁLCULO DAS PRIORIDADES GLOBAIS}

Após obter a prioridade média local de cada critério à luz do Foco Principal $\left(P M L_{c}\right)$, de cada subcritério à luz dos respectivos critérios $\left(P M L_{s}\right)$ e de cada alternativa $\left(P M L_{a} \dot{i}\right.$ à luz de cada subcritério, a prioridade global $\left(P G_{a_{i}}\right)$ de um Centro Acadêmico $i$ pode ser obtida por meio da Equação 3.

$$
P G_{a_{i}}=\left(P M L_{C_{1}} \times P M L_{a_{i}}\right)+\left(P M L_{C_{2}} \times P M L_{a_{i}}\right)+\ldots+\left(P M L_{C_{n}} \times P M L_{a_{i}}\right)
$$

Para fins de cálculo, ressalta-se que, para a computação do $P M L_{C}$, deve-se considerar as prioridades médias locais dos $m$ subcritérios, relacionados ao seu respectivo critério $n$, como descrito na Equação 4.

$$
P M L_{C_{n}}=\left(P M L_{c_{n}} \times P M L_{s_{1}}\right)+\left(P M L_{c_{n}} \times P M L_{s_{2}}\right)+\ldots+\left(P M L_{c_{n}} \times P M L_{s_{m}}\right)
$$

Os resultados encontram-se na Tabela 6, sendo possível constatar que, ao considerar as PGs, o Centro de Ciência e Tecnologia (CCT), da IES em estudo, apresenta maior nível de criticidade, uma vez que, em termos de melhoria da qualidade, a maior importância deve 
ser dada aos critérios de pior desempenho da QVT.

Tabela 6 - Hierarquia das alternativas à luz de todos os critérios

\begin{tabular}{|c|c|c|c|c|c|c|c|c|}
\hline & & & & CCT & CCTA & $\mathrm{CCH}$ & CBB & REITORIA \\
\hline Critério & Subcritério & $P M L_{c}$ & $P M L_{s}$ & \multicolumn{5}{|c|}{$P M L_{a}$} \\
\hline \multirow{7}{*}{$\overline{0}$} & $\mathrm{P} 1$ & \multirow{7}{*}{ 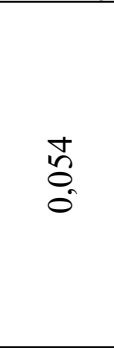 } & 0,084 & 0,261 & 0,241 & 0,111 & 0,283 & 0,103 \\
\hline & P2 & & 0,094 & 0,200 & 0,200 & 0,200 & 0,200 & 0,200 \\
\hline & P3 & & 0,084 & 0,250 & 0,250 & 0,125 & 0,250 & 0,125 \\
\hline & P4 & & 0,149 & 0,254 & 0,197 & 0,147 & 0,254 & 0,147 \\
\hline & P5 & & 0,178 & 0,414 & 0,177 & 0,143 & 0,163 & 0,103 \\
\hline & P6 & & 0,178 & 0,410 & 0,163 & 0,123 & 0,202 & 0,101 \\
\hline & P7 & & 0,235 & 0,364 & 0,182 & 0,182 & 0,182 & 0,091 \\
\hline \multirow{7}{*}{ ลี } & P8 & \multirow{7}{*}{$\begin{array}{l}\infty \\
0 \\
0 \\
0\end{array}$} & 0,089 & 0,198 & 0,198 & 0,176 & 0,231 & 0,198 \\
\hline & P9 & & 0,219 & 0,166 & 0,116 & 0,219 & 0,189 & 0,311 \\
\hline & P10 & & 0,125 & 0,197 & 0,254 & 0,147 & 0,254 & 0,147 \\
\hline & P11 & & 0,125 & 0,200 & 0,200 & 0,200 & 0,200 & 0,200 \\
\hline & $\mathrm{P} 12$ & & 0,152 & 0,200 & 0,200 & 0,200 & 0,200 & 0,200 \\
\hline & P13 & & 0,137 & 0,309 & 0,167 & 0,132 & 0,142 & 0,250 \\
\hline & $\mathrm{P} 14$ & & 0,152 & 0,380 & 0,149 & 0,161 & 0,149 & 0,161 \\
\hline \multirow{5}{*}{$\tilde{\theta}$} & P15 & \multirow{5}{*}{$\frac{n}{0}$} & 0,162 & 0,466 & 0,181 & 0,080 & 0,198 & 0,075 \\
\hline & P16 & & 0,117 & 0,381 & 0,095 & 0,126 & 0,178 & 0,221 \\
\hline & P17 & & 0,140 & 0,301 & 0,089 & 0,186 & 0,160 & 0,265 \\
\hline & P18 & & 0,442 & 0,405 & 0,248 & 0,059 & 0,144 & 0,144 \\
\hline & P19 & & 0,140 & 0,315 & 0,315 & 0,100 & 0,156 & 0,115 \\
\hline \multirow{2}{*}{$\overrightarrow{0}$} & P20 & ह & 0,250 & 0,421 & 0,158 & 0,076 & 0,187 & 0,158 \\
\hline & $\mathrm{P} 21$ & 0 & 0,750 & 0,477 & 0,112 & 0,127 & 0,127 & 0,157 \\
\hline \multirow{2}{*}{ ñ } & P22 & \multirow{2}{*}{$\begin{array}{l}\infty \\
0 \\
0 \\
0\end{array}$} & 0,333 & 0,404 & 0,159 & 0,146 & 0,146 & 0,146 \\
\hline & $\mathrm{P} 23$ & & 0,667 & 0,406 & 0,121 & 0,121 & 0,164 & 0,188 \\
\hline \multirow{3}{*}{ ஜ̊ } & P24 & \multirow{3}{*}{$\begin{array}{l}\stackrel{\infty}{\infty} \\
\stackrel{+}{0} \\
0\end{array}$} & 0,200 & 0,200 & 0,200 & 0,200 & 0,200 & 0,200 \\
\hline & P25 & & 0,400 & 0,200 & 0,200 & 0,200 & 0,200 & 0,200 \\
\hline & P26 & & 0,400 & 0,197 & 0,197 & 0,172 & 0,263 & 0,172 \\
\hline \multirow{2}{*}{$\hat{\theta}$} & $\mathrm{P} 27$ & \multirow{2}{*}{ क̂ } & 0,500 & 0,198 & 0,198 & 0,231 & 0,176 & 0,198 \\
\hline & P28 & & 0,500 & 0,375 & 0,186 & 0,130 & 0,161 & 0,148 \\
\hline \multicolumn{4}{|c|}{$P G^{\prime} s$} & 0,294 & 0,182 & 0,156 & 0,193 & 0,175 \\
\hline
\end{tabular}

\subsection{VERIFICAÇÃO DA CONSISTÊNCIA DOS JULGAMENTOS}

De acordo com Saaty (2000), o método AHP permite determinar o grau de inconsistência dos julgamentos paritários avaliando a intensidade com que o autovalor da matriz de julgamentos se afasta de sua ordem. Para a execução dos cálculos, o pesquisador propôs um procedimento composto de algumas etapas, ilustradas por meio da Matriz de Critérios-Foco Principal:

a) Construção de uma matriz auxiliar A' A' com os valores da matriz de julgamento multiplicado pelas PML's de cada critério: 
$A^{\prime}=\left(\begin{array}{ccccccc}1 \times 0,054 & 1 \times 0,058 & 1 / 4 \times 0,159 & 1 / 3 \times 0,159 & 1 \times 0,058 & 1 / 8 \times 0,480 & 2 \times 0,033 \\ 1 \times 0,054 & 1 \times 0,058 & 1 / 3 \times 0,159 & 1 / 3 \times 0,159 & 1 \times 0,058 & 1 / 7 \times 0,480 & 2 \times 0,033 \\ 4 \times 0,054 & 3 \times 0,058 & 1 \times 0,159 & 1 \times 0,159 & 3 \times 0,058 & 1 / 5 \times 0,480 & 5 \times 0,033 \\ 4 \times 0,054 & 3 \times 0,058 & 1 \times 0,159 & 1 \times 0,159 & 3 \times 0,058 & 1 / 5 \times 0,480 & 5 \times 0,033 \\ 1 \times 0,054 & 1 \times 0,058 & 1 / 3 \times 0,159 & 1 / 3 \times 0,159 & 1 \times 0,058 & 1 / 7 \times 0,480 & 2 \times 0,033 \\ 8 \times 0,054 & 7 \times 0,058 & 5 \times 0,159 & 5 \times 0,159 & 7 \times 0,058 & 1 \times 0,480 & 9 \times 0,033 \\ 1 / 2 \times 0,054 & 1 / 2 \times 0,058 & 1 / 5 \times 0,159 & 1 / 5 \times 0,159 & 1 / 2 \times 0,058 & 1 / 9 \times 0,480 & 1 \times 0,033\end{array}\right)=\left(\begin{array}{l}0,0 \\ 0,0 \\ 0,2 \\ 0,2 \\ 0,0 \\ 0,4 \\ 0,0\end{array}\right.$

b) Soma dos elementos de cada linha da matriz auxiliar A' $A^{\prime} A^{\prime}$, constituindo o vetor de prioridade $\mathrm{P}^{\prime} \mathrm{P}^{\prime}$. Os valores resultantes serão divididos pelo valor da PML associada a esta linha, constituindo os elementos do vetor $\mathrm{P}_{\text {aux }} P_{\text {aux }}$. Os vetores obtidos são:

$$
\begin{gathered}
P^{\prime}=(0,375 ; 0,410 ; 1,142 ; 1,142 ; 0,410 ; 3,607 ; 0,235) \\
P_{\text {aux }}=\left(\frac{0,375}{0,054} ; \frac{0,410}{0,058} ; \frac{1,142}{0,159} ; \frac{1,142}{0,159} ; \frac{0,410}{0,058} ; \frac{3,607}{0,480} ; \frac{0,235}{0,033}\right)=i(6,985 ; 7,076 ; 7,191 ; 7,191 ; 7,0
\end{gathered}
$$

c) Cálculo do autovalor máximo por meio da média dos elementos de $\mathrm{P}_{\text {aux }} P_{a u x} P_{\text {aux }}$ :

$$
\lambda_{\text {máx }}=7,161 \text {. }
$$

d) Cálculo do Índice de Consistência (ICIC) e da Razão de Consistência (RCRC), sendo IRIR um índice de consistência para uma matriz recíproca, com elementos não negativos e gerada randomicamente. Para uma matriz de julgamentos de ordem 7, o valor de IRIR é 1,32. Segundo Saaty (2000) os julgamentos de uma matriz são consistentes quando $\mathrm{RC} \leq 0,10 R C \leq 0,10$.

$$
\begin{gathered}
I C=\frac{\lambda_{\text {máx }}-n}{n-1}=\frac{7,161-7}{6}=0,027 \\
R C=\frac{I C}{I R}=\frac{0,027}{1,32}=0,020
\end{gathered}
$$

Os valores de RCRC para as demais matrizes de julgamento são apresentadas nas Tabelas 7 e 8, sendo todas consideradas consistentes.

Tabela 7 - Razão de Consistência (RC) à luz dos critérios

\begin{tabular}{cccccccc}
\hline Matriz & D1 & D2 & D3 & D4 & D5 & D6 & D7 \\
\hline RC & 0,011 & 0,017 & 0,012 & 0,000 & 0,000 & 0,000 & 0,000 \\
\hline \multicolumn{7}{c}{ Fonte: autoria própria (2019) }
\end{tabular}

Tabela 8 - Razão de Consistência (RC) à luz dos subcritérios

\begin{tabular}{cccccccc}
\hline Matriz & P1 & P2 & P3 & P4 & P5 & P6 & P7 \\
\hline RC & 0,007 & 0,000 & 0,000 & 0,017 & 0,014 & 0,013 & 0,000 \\
\hline
\end{tabular}




\begin{tabular}{cccccccc}
\hline Matriz & P8 & P9 & P10 & P11 & P12 & P13 & P14 \\
\hline RC & 0,013 & 0,021 & 0,017 & 0,000 & 0,000 & 0,017 & 0,006 \\
\hline Matriz & P15 & P16 & P17 & P18 & P19 & P20 & P21 \\
\hline RC & 0,013 & 0,016 & 0,012 & 0,013 & 0,009 & 0,007 & 0,012 \\
\hline Matriz & P22 & P23 & P24 & P25 & P26 & P27 & P28 \\
\hline RC & 0,004 & 0,058 & 0,000 & 0,000 & 0,017 & 0,013 & 0,014 \\
\hline
\end{tabular}

Fonte: autoria própria (2019)

\section{CONSIDERAÇÕES FINAIS}

A avaliação e análise da QVT justifica-se para o indivíduo e para a organização, pela importância da identificação dos pontos falhos, valorização dos fatores essenciais para uma apropriada qualidade de vida e a partir de então, traçar estratégias de melhoria na satisfação dos colaboradores com o trabalho, conduzindo assim a maior produtividade e, consequentemente, contentamento dos clientes. A partir do momento em que as instituições possuem estas informações, podem então melhor direcionar e fundamentar programas de promoção da QVT dos colaboradores, proporcionando melhores condições de trabalho ao indivíduo, oferecendo-lhe a oportunidade de empregar todo o seu potencial produtivo.

Desejando contribuir para análise desta problemática, no presente artigo foi realizado um estudo experimental com o objetivo de identificar o Centro Acadêmico crítico em termo do grau desempenho da QVT na Universidade, segundo na percepção dos servidores técnico-administrativos. Para tanto, foi aplicado um instrumento de avaliação composto por múltiplos critérios e o emprego do método AHP.

Como resultados, foi possível identificar, um indicativo de criticidade, em termos de QVT no Centro de Ciência e Tecnologia, da IES em questão. Essa avaliação sugere que este centro acadêmico deve ser priorizado em prol da melhoria da qualidade de vida na IES. Da mesma forma, através dos julgamentos paritários em relação ao foco principal, foi possível observar que a dimensão Compensação Justa e Adequada (D6), é o critério de maior preocupação, quanto ao grau de desempenho da QVT. Vale destacar que, todas as matrizes de julgamentos foram consideradas consistentes.

Diante do exposto, na percepção dos funcionários técnicos-administrativos, as ações prioritariamente necessárias para melhorar a QVT na IES, em especial o CCT, devem estar focadas em: (i) manter os salários compatíveis com os salários pagos em outras organizações; (ii) oferecer salários compatíveis com o esforço, experiência, habilidade e qualificação das atividades desempenhadas; e (iii) conceder os benefícios previstos em lei e realizar exames médicos periódicos.

\section{REFERÊNCIAS BIBLIOGRÁFICAS}

[1] COSTA, H. G. Introdução ao Método de Análise Hierárquica - Análise Multicritério no Auxílio à Decisão. Niterói, Biblioteca da Escola de Engenharia e Instituto de Computação da UFF, 2002.

[2] COSTA, H. G. IPÊ 1.0: Guia do Usuário. Niterói: UFF, 2004. Disponível em $<$ http://www.producao.uff.br/conteudo/rpep/volume42004/RelPesq_V4_2004_05.pdf> . Acesso em 04 mai. 2017.

[3] DAVIS, K.; WERTHER, W. B. Administração de pessoal e recursos humanos. McGraw Hill São Paulo, 1983. 
[4] ENTRINGER, T. C.; FREITAS, A. L. P. Qualidade de vida no trabalho do técnicoadministrativo: um estudo no centro de ciências e tecnologia de uma universidade. In: ENEGEP, 34, 2005, Curitiba. Anais... Rio de Janeiro: ABEPRO, 2014a.

[5] ENTRINGER, T. C.; FREITAS, A. L. P. Mensuração da Qualidade de vida no trabalho do técnico-administrativo: um estudo no centro de ciências e tecnologia de uma universidade. In: Simpósio de Engenharia de Produção, 21, Anais... Bauru: SIMPEP, 2014b.

[6] ENTRINGER, T. C.; FREITAS, A. L. P. Qualidade de vida no trabalho do servidor técnico-administrativo: um estudo no Centro de Ciências e Tecnologias Agropecuárias de uma IES pública. In: ENEGEP, 35, 2015, Fortaleza. Anais... Rio de Janeiro: ABEPRO, 2015.

[7] FREITAS, A. L. P.; SOUZA, R. G. B. Um modelo para avaliação da qualidade de vida no trabalho em universidades públicas. Sistemas \& Gestão, v. 4, p. 136-154, 2009.

[8] FREITAS, A. L. P.; SOUZA, R. G. B.; QUINTELLA, H. L. M. M. Qualidade de vida no trabalho do técnico-administrativo em IES públicas: uma análise exploratória. Revista brasileira de Qualidade de Vida. v.5, n.2, p.1-12, 2013.

[9] GOYAL, P.; RAHMAN, Z.; KAZMI, A. A. Identification and prioritization of corporate sustainability practices using analytical hierarchy process. Journal of Modelling in Management, 10(1), 23-49, 2015.

[10] HACKMAN, J. R.; OLDHAM, G.R. Development of the job diagnostic survey. Journal of Applied Psychology. v. 60, n. 2, p. 159-170, 1975.

[11] JANES, P.; WISNOM, M. Changes in tourism industry quality of work life practices. Journal of Tourism Insights. v. 1, n. 1, p. 13. 2011.

[12] KAVURMACI, M.; ÜSTÜN, A. K. Assessment of groundwater quality using DEA and AHP: a case study in the Sereflikochisar region in Turkey. Environmental Monitoring and Assessment, v. 188, n. 4, abr. 2016.

[13] KEREN, B.; HADAD, Y. ABC Inventory Classification Using AHP and Ranking Methods via DEA. IEEE, fev. 2016

[14] LAÑEZ, M. M.; CUNHA, C. B. da. Uma metodologia para a seleção de um provedor de serviços logísticos. Produção, São Paulo, v. 16, n. 3, p.394-412, 2006.

[15] MANJUNATH, S. J.; JURIAN, S. Impact of quality work life of the hotel employees in customer satisfaction - a study on star hotels in Bangalore. South Asian Journal of Tourism and Heritage. v. 4, n. 2, p. 78-92. 2011.

[16] MIRKAMALI, S. M.; THANI, F. N. Study on the quality of work life (QWL) among faculty members of University of Tehran (UT) and Sharif University of Technology (SUT). Procedia - Social and Behavioral Sciences, v. 29, p. 179-187. 2011.

[17] PINTO, L. B. Qualidade de vida no trabalho para os servidores técnico-administrativos de uma Universidade Federal em Minas Gerais. Dissertação, 173 f (Pós-graduação em Organizações, Gestão e Sociedade) - Universidade Federal de Lavras, 2013.

[18] SAATY, T. L. How to make a decision: the Analytic Hierarchy Process. European Journal of Operations Research 48, p.9-26, 1990.

[19] SAATY, T. L. Decision making for leaders. Pittsburg, USA: WS. Publications, 2000. 
[20] SINGH, A.; PRASHER, A. Measuring healthcare service quality from patients' perspective: using Fuzzy AHP application. Total Quality Management \& Business Excellence, p. 1-17, 22 mar. 2017.

[21] THANASSOULIS, E. et al. Evaluating higher education teaching performance using combined analytic hierarchy process and data envelopment analysis. Journal of the Operational Research Society, v. 68, n. 4, p. 431-445, 1 abr. 2017.

[22] TOKEN, B. Job satisfaction of academy staff: an empirical study on Turkey. Quality Assurance in Education, vol. 19, n. 2, p. 156-169, 2011.

[23] TREVIZANO, W. A.; FREITAS, A. L. P. Emprego do Método da Análise Hierárquica (AHP) na seleção de processadores. In: ENEGEP, 25., 2005, Porto Alegre. Anais.... Rio de Janeiro: Abepro, 2005. p. 2972 - 2979.

[24] VELASCO, M. H. E.; FREITAS, A. L. P.. Emprego de métodos de apoio multicritério à decisão para seleção de uma escola de idiomas. Revista Produção Online, Florianópolis, SC, v.14, n. 4, p. 1433-1451, out./dez. 2014.

[25] WALTON, R. E. Quality of working life: what is it? Sloan Management Review, v.15, n. 1, p. 11-21, 1973.

[26] WESTLEY, W. A. Problems and solutions in the quality of working life. Human Relations. v.32, p.113-123. 1979. 